\title{
Native incretins prevent the development of atherosclerotic lesions in apolipoprotein $\mathbf{E}$ knockout mice
}

\author{
M. Nagashima • T. Watanabe $\cdot$ M. Terasaki • \\ M. Tomoyasu • K. Nohtomi • J. Kim-Kaneyama • \\ A. Miyazaki • T. Hirano
}

Received: 2 March 2011 / Accepted: 31 May 2011 /Published online: 24 July 2011

(C) The Author(s) 2011. This article is published with open access at Springerlink.com

\begin{abstract}
Aims/hypothesis Several lines of evidence suggest that incretin-based therapies suppress the development of cardiovascular disease in type 2 diabetes. We investigated the possibility that glucagon-like peptide-1 (GLP-1) and glucose-dependent insulinotropic polypeptide (GIP) can prevent the development of atherosclerosis in $\mathrm{Apoe}^{-/-}$mice. Methods Apoe ${ }^{-/-}$mice (17 weeks old) were administered GLP-1(7-36)amide, GLP-1(9-36)amide, GIP(1-42) or GIP (3-42) for 4 weeks. Aortic atherosclerosis, oxidised LDLinduced foam cell formation and related gene expression in exudate peritoneal macrophages were determined.

Results Administration of GLP-1(7-36)amide or GIP(142) significantly suppressed atherosclerotic lesions and macrophage infiltration in the aortic wall, compared with vehicle controls. These effects were cancelled by co-
\end{abstract}

M. Nagashima and T. Watanabe contributed equally to this study.

Electronic supplementary material The online version of this article (doi:10.1007/s00125-011-2241-2) contains peer-reviewed but unedited supplementary material, which is available to authorised users.

M. Nagashima $\cdot$ M. Terasaki $\cdot$ M. Tomoyasu $\cdot$ K. Nohtomi ·

T. Hirano $(\square)$

Department of Medicine, Division of Diabetes, Metabolism,

and Endocrinology, Showa University School of Medicine,

1-5-8 Hatanodai, Shinagawa-ku,

Tokyo 142-8666, Japan

e-mail: hirano@med.showa-u.ac.jp

J. Kim-Kaneyama • A. Miyazaki

Department of Biochemistry,

Showa University School of Medicine,

Tokyo, Japan

T. Watanabe

Laboratory of Cardiovascular Medicine,

Tokyo University of Pharmacy and Life Sciences,

Tokyo, Japan infusion with specific antagonists for GLP-1 and GIP receptors, namely exendin(9-39) or $\mathrm{Pro}^{3}(\mathrm{GIP})$. The antiatherosclerotic effects of GLP-1(7-36)amide and GIP(142) were associated with significant decreases in foam cell formation and downregulation of CD36 and acyl-coenzyme A:cholesterol acyltransferase-1 (ACAT-1) in macrophages. GLP-1 and GIP receptors were both detected in Apoe ${ }^{-/}$ mouse macrophages. Ex vivo incubation of macrophages with GLP-1(7-36)amide or GIP(1-42) for $48 \mathrm{~h}$ significantly suppressed foam cell formation. This effect was wholly abolished in macrophages pretreated with exendin(9-39) or $\left(\mathrm{Pro}^{3}\right)$ GIP, or with an adenylate cyclase inhibitor, MDL12,330A, and was mimicked by incubation with an adenylate cyclase activator, forskolin. The inactive forms, GLP-1(9-36)amide and GIP(3-42), had no effects on atherosclerosis and macrophage foam cell formation.

Conclusions/interpretation Our study is the first to demonstrate that active forms of GLP-1 and GIP exert antiatherogenic effects by suppressing macrophage foam cell formation via their own receptors, followed by cAMP activation. Molecular mechanisms underlying these effects are associated with the downregulation of CD36 and ACAT-1 by incretins.

Keywords Atherosclerosis · Cholesterol · Dipeptidyl peptidase-4 - Glucagon-like peptide-1 - Glucose-dependent insulinotropic polypeptide · Incretin · Macrophages .

Mouse model

$\begin{array}{ll}\text { Abbreviations } \\ \text { ABC } & \text { ATP-binding cassette transporter } \\ \text { ACAT-1 } & \text { Acyl-coenzyme A:cholesterol acyltransferase-1 } \\ \text { DPP-4 } & \text { Dipeptidyl peptidase-4 } \\ \text { ERK } & \text { Extracellular signal-regulated kinase } \\ \text { GIP } & \text { Glucose-dependent insulinotropic polypeptide }\end{array}$




$\begin{array}{ll}\text { GIPR } & \text { GIP receptor } \\ \text { GLP-1 } & \text { Glucagon-like peptide-1 } \\ \text { GLP-1R } & \text { GLP-1 receptor } \\ \text { PI3K } & \text { Phosphatidylinositol-3-kinase } \\ \text { PKA } & \text { Protein kinase A } \\ \text { PKC } & \text { Protein kinase C } \\ \text { SMC } & \text { Smooth muscle cell } \\ \text { SR-BI } & \text { Scavenger receptor class B type I }\end{array}$

\section{Introduction}

The incretins, such as glucagon-like peptide-1 (GLP-1) and glucose-dependent insulinotropic polypeptide (GIP), are secreted postprandially from the L-cells of the lower gut and K-cells of the upper gut, respectively, to regulate glucose homeostasis [1]. New treatments based on incretin hormones have provided a novel way of addressing parts of the complex pathophysiology of type 2 diabetes [1]. Type 2 diabetes is well known to accelerate the clinical course of atherosclerosis, a condition associated with arterial endothelial dysfunction and several metabolic abnormalities. Incretin-based therapies have been reported to decrease blood pressure and atherogenic lipoproteins, and to improve vascular inflammation and endothelial dysfunction [1-3]. The vasoprotective properties of GLP-1 led us to speculate that treatment with GLP-1 can prevent the development of atherosclerosis through GLP-1 receptors (GLP-1R). The effect of GIP on the cardiovascular system is largely unknown, although GIP receptors (GIPR) have been detected in vascular tissues [4].

Both GLP-1 and GIP are rapidly metabolised via enzymatic degradation by the dipeptidyl peptidase-4 (DPP-4) [2]. The DPP-4-resistant GLP-1R agonists (incretin mimetics) and DPP-4 inhibitors are used as therapeutic agents to enhance the action of incretin [2]. Exendin-4, a GLP-1R agonist, suppresses monocytic adhesion to the aortic wall and aortic smooth muscle cell (SMC) proliferation [5, 6]. Liraglutide, a long-acting GLP-1R agonist, and vildagliptin, a DPP-4 inhibitor, attenuate production of plasminogen activator inhibitor 1 (PAI-1) and vascular cell adhesion molecule-1 (VCAM-1) in vascular cells [7, 8].

The development of atherosclerosis is influenced by abnormalities in cellular cholesterol homeostasis in subendothelial macrophages. The level of non-esterified cholesterol within cells is increased by the uptake of oxidised LDL via CD36 [9]. It is decreased by the efflux of non-esterified cholesterol mediated by ATP-binding cassette transporter $(\mathrm{ABC}) \mathrm{A} 1, \mathrm{ABCG} 1$ or scavenger receptor class B type I (SR-BI) [10]. To protect cells from the toxicity that would result from excessive non-esterified cholesterol accumulation, the non-esterified cholesterol is esterified to cholesteryl ester by acyl-coenzyme A:cholesterol acyltransferase-1 (ACAT-1) [9]. ACAT-1 thereby promotes cholesteryl ester accumulation in macrophages, which contributes to foam cell formation.

The present study clarified for the first time the preventive effects of native GLP-1 and GIP on the development of aortic atherosclerotic lesions in $A p o e^{-/}$ mice. To explore the cellular and molecular mechanisms underlying the anti-atherosclerotic actions, we assessed the comprehensive effects of these incretins on macrophage foam cell formation, aortic SMC proliferation and gene expression of proatherogenic cytokines in aortic endothelial cells.

\section{Methods}

Chemicals and reagents Human GLP-1(7-36)amide, GIP (1-42), GIP(3-42) and exendin(9-39), a GLP-1R antagonist, were purchased from AnaSpec (San Jose, CA, USA). Human GLP-1(9-36)amide and $\left(\right.$ Pro $\left.^{3}\right)$ GIP, a GIPR antagonist, were purchased from Bachem (Torrance, CA, USA) and Abgent (San Diego, CA, USA), respectively. Vildagliptin analogue (PKF275-055) was a generous gift from Novartis (Basel, Switzerland). MDL12,330A and forskolin were purchased from Sigma (St. Louis, MO, USA).

Animal experiments Animal experiments were performed in accordance with the NIH guidelines for the Care and Use of Laboratory Animals and were approved by the Institutional Animal Care and Use Committee of Showa University. A total of 346 male Apoe $^{-/-}$mice were purchased at the age of 8 weeks from Sankyo Labo Service (Tokyo, Japan) and kept on normal chow until the age of 17 weeks. From 17 weeks of age, nine groups of Apoe ${ }^{-/-}$mice were started to be respectively infused with GLP-1(7-36)amide, GLP-1(9-36)amide (both $2.2 \mathrm{nmol} \mathrm{kg}^{-1} \mathrm{day}^{-1}$ ) [11], exendin(9-39) (22 $\left.\mathrm{nmol} \mathrm{kg}^{-1} \mathrm{day}^{-1}\right)$, GLP-1(7-36)amide +exendin(9-39) (the same doses), GIP(1-42), GIP(3-42), $\left(\right.$ Pro $\left.^{3}\right)$ GIP, GIP $(1-42)+\left(\right.$ Pro $\left.^{3}\right)$ GIP (all $25 \mathrm{nmol} \mathrm{kg}^{-1}$ day $^{-1}$ ) [12] and saline (vehicle). Infusion was for 4 weeks by osmotic mini-pumps (Alzet Model 1007D; Durect, Cupertino, CA, USA). An atherogenic diet containing 30\% fat, $20 \%$ sucrose and $8 \% \mathrm{NaCl}$ (Oriental Yeast, Tokyo, Japan) [13], and continuous administration of incretins were started at the same time.

Animal measurements Four weeks after infusion into Apoe $^{-/-}$mice, systolic blood pressure was measured using indirect tail-cuff equipment (MK-2000; Muromachi Kikai, Tokyo, Japan) [14]. Blood samples were collected after $6 \mathrm{~h}$ fast. Plasma concentrations of glucose, total cholesterol, HDL-cholesterol, triacylglycerol and NEFA were measured 
by enzymatic methods using an autoanalyser (Hitachi 7020; Hitachi, Tokyo, Japan) [14]. Plasma concentrations of active GLP-1, total GIP and insulin were determined by ELISA (Active GLP-1 ELISA Kit, Millipore, Billerica, MA, USA; GIP Assay Kit, Immuno-Biological Laboratories, Maebashi, Japan; Ultra Sensitive PLUS Mouse Insulin ELISA Kit, Morinaga, Yokohama, Japan).

Atherosclerotic lesion assessment At the ages of 17 and 21 weeks (before and 4 weeks after infusion), the $A p o e^{-/-}$ mice were killed by anaesthetisation with diethyl ether. The whole aorta was washed with perfused PBS and fixed with $4 \%$ paraformaldehyde (wt/vol.) [14]. The aorta was excised from the root to the abdominal area, and the connective and adipose tissues carefully removed. The entire aorta and cross-sections of the aortic root were stained with oil red $\mathrm{O}$ for assessment of atherosclerotic lesions [14]. Macrophage infiltration into the aortic wall was visualised by anti-mouse MOMA-2 antibody staining [10, 14]. Haematoxylin was used for nuclear staining. The areas of the aorta with atherosclerotic lesions were traced by an investigator blind to the treatment and measured by an image analyser (Adobe Photoshop, San Jose, CA; NIH Scion Image, Frederick, MD, USA) [10, 14]. The severity of atheromatous plaques and degree of macrophage accumulation were expressed as percentages of the lesion area relative to the entire crosssection of the aortic wall $[10,14]$.

Cell culture Exudate peritoneal cells were isolated from treated Apoe $^{-/-}$mice at 21 weeks of age by peritoneal lavage with $8 \mathrm{ml}$ ice-cold PBS 4 days after intraperitoneal injection of $4 \mathrm{ml}$ aged-autoclaved thioglycolate broth [13, 14]. The cells were suspended in culture medium (RPMI1640 supplemented with $10 \%$ FCS, streptomycin and penicillin) and seeded on to 6 -cm dishes $\left(4 \times 10^{6}\right.$ cells $\left[2 \mathrm{ml}^{-1} \mathrm{dish}^{-1}\right.$ ) for western blotting analysis or RT-PCR and $3.5-\mathrm{cm}$ dishes $\left(3 \times 10^{6}\right.$ cells $\left.[1 \mathrm{ml}]^{-1} \mathrm{dish}^{-1}\right)$ for cholesterol esterification assay. After $1 \mathrm{~h}$ incubation at $37^{\circ} \mathrm{C}$ in $5 \% \mathrm{CO}_{2}$ to allow adhesion, the medium was discarded to remove non-adherent cells $[13,14]$.

Western blotting analysis Adherent macrophages were extracted with $80 \mu 1$ 10\% SDS (wt/vol.) [10, 14]. Cell protein $(30 \mu \mathrm{g}$ aliquots) was separated by $10 \%$ SDS-PAGE and subjected to immunoblotting with the following antibodies: rabbit polyclonal antibody against human ACAT-1 (Dartmouth Medical School, Hanover, NH, USA), GLP-1R (Abcam, Tokyo, Japan), ABCA1 and ABCG1, or against mouse SR-BI (Novus Biologicals, Littleton, CO, USA); goat polyclonal antibody against mouse CD36 (R\&D Systems, Minneapolis, MN, USA) or rat GIPR (Santa Cruz Biotechnology, Santa Cruz, CA, USA); and mouse monoclonal anti- $\beta$-actin antibody (Sigma). The densities of the bands were measured using Light-Capture and Densitograph software (AE-6962FC, CS Analyser version 2.0; ATTO, Tokyo, Japan) [10, 14].

Cholesterol esterification assay Adherent macrophages were incubated for $18 \mathrm{~h}$ with culture medium containing $10 \mu \mathrm{g} / \mathrm{ml}$ human oxidised LDL in the presence of $0.1 \mathrm{mmol} / 1\left[{ }^{3} \mathrm{H}\right]$ oleate conjugated with BSA $[13,14]$. Cellular lipids were extracted and the radioactivity of cholesterol $\left[{ }^{3} \mathrm{H}\right]$ oleate was determined by thin-layer chromatography $[13,14]$.

Confocal microscopy Macrophages seeded on to Lab-Tek Chamber Slide (Thermo Fisher Scientific, Waltham, MA, USA) were fixed with $4 \%$ paraformaldehyde (wt/vol.) and stained with rabbit polyclonal anti-GLP-1R antibody (Abcam) or goat polyclonal anti-GIPR antibody (Santa Cruz Biotechnology), followed by anti-rabbit or anti-goat Alexa Fluor 488 (Invitrogen, Carlsbad, CA, USA). Nuclei were visualised using propidium iodide. Fluorescencestained cells were examined on confocal microscope (Radiance 2100; Bio Rad, Hercules, CA, USA). Fluorescence was detected with wavelengths for excitation at $488 \mathrm{~nm}$ (Alexa Fluor 488) and $543 \mathrm{~nm}$ (propidium iodide).

Real-time RT-PCR Pre-designed TaqMan probe sets of Glp1r, Gipr and 18S rRNA were purchased from Applied Biosystems (Carlsbad, CA, USA). Total RNA was extracted using a reagent (ISOGEN; Nippon-Gene, Tokyo, Japan) from exudate peritoneal macrophages, and from brain, liver, pancreas, aorta and adipose tissues of non-treated $\mathrm{Apoe}^{-/-}$ mice at 9 weeks of age. cDNA was synthesised from isolated RNA templates with a kit (High-Capacity cDNA Archive Kit; Applied Biosystems). Real-time PCR was performed using TaqMan Gene Expression Assays (Applied Biosystems). Amplification and fluorescent measurements were carried out during the elongation step with a sequence detection system (ABI PRISM 7700; Applied Biosystems).

Measurements human GLP-1R and GIPR The investigation was approved by the Ethics Committee of Showa University. Informed consent was obtained from all participants. Human peripheral mononuclear cells were isolated from the blood of six healthy volunteers (four men, two women, age $25 \pm 2$ years) as described previously [10, 15]. Monocytes purified using anti-CD14 antibody-conjugated magnetic microbeads (Miltenyi Biotec, Auburn CA, USA) were seeded on to $6-\mathrm{cm}$ dishes $\left(4 \times 10^{6}\right.$ cells $\left[2 \mathrm{ml}^{-1} \mathrm{dish}^{-1}\right)$ and incubated in RPMI-1640 containing 10\% human serum for monocytic differentiation into macrophages. Total RNA was extracted from the monocytes after $1 \mathrm{~h}$ and macrophages collected 7 days after the incubation, after which GIPR mRNA levels were quantified by real-time RT-PCR. 
THP1 cells (ATCC, Manassas, VA, USA) were suspended in culture medium and seeded on to $6-\mathrm{cm}$ dishes $\left(4 \times 10^{6}\right.$ cells $\left[2 \mathrm{ml}^{-1} \mathrm{dish}^{-1}\right)$. To induce differentiation into macrophages, THP1 cells were incubated for $48 \mathrm{~h}$ in the presence of $100 \mathrm{nmol} / \mathrm{l}$ phorbol myristate acetate in RPMI1640 containing $10 \%$ FCS. Total RNA was extracted from the cells after $1 \mathrm{~h}$ and $48 \mathrm{~h}$ after start of incubation, and $G L P 1 R$ and GIPR mRNA levels were quantified by realtime RT-PCR.

Gene expression of proatherogenic cytokines in aortic endothelial cells Human aortic endothelial cells (Lonza, Walkersville, MD, USA) in passages 4 to 6 were seeded on to 12 -well plates and grown to semi-confluence in $1 \mathrm{ml}$ EGM-2 involving EGM-2 Single Quots (Lonza). The medium was then replaced with $1 \mathrm{ml}$ serum-free RPMI1640 with the indicated concentrations of GLP-1(7-36) amide or GIP(1-42) for $2 \mathrm{~h}$. We determined mRNA levels of MCP1 (also known as CCL2), VCAM1, ICAM1 and PAI1 (also known as SERPINE1) in endothelial cells by real-time RT-PCR.

Aortic SMC proliferation assay Human aortic SMCs (Lonza) were seeded on to 6 -well plates $\left(3 \times 10^{4}\right.$ cells $\left[1 \mathrm{ml}^{-1}\right.$ well $^{-1}$ ) and cultured for $24 \mathrm{~h}$ in SmBM (Lonza) containing 5\% FCS. The cells were incubated for $29 \mathrm{~h}$ with GLP-1(7-36)amide or GIP(1-42) along with PKF275-055 in order to prevent degradation of incretins, as DDP-4 activity is far higher in aortic SMCs than in aortic endothelial cells [16]. The cells were exposed to BrdU $(10 \mu \mathrm{mol} / \mathrm{l})$ for the last $24 \mathrm{~h}$. BrdU-positive cells were visualised by immunostaining and determined under the microscope.

Statistical analysis All values are expressed as mean \pm SEM. Data were compared by two-tailed unpaired Student's $t$ test between two groups and by one-way ANOVA followed by Bonferroni's post hoc test among three or more groups, using Statview-J 5.0 (SAS Institute, Cary, NC, USA). Differences were considered statistically significant at $p<0.05$.

\section{Results}

Atherosclerotic lesions In 17-week-old Apoe $e^{-/-}$mice, mild atherosclerotic lesions were observed in the proximal portion of the aorta (Fig. 1a, g, m). Atherosclerotic lesions were significantly developed in 21-week-old $\mathrm{Apoe}^{-1-}$ mice (Fig. 1b, h, n). Infusion of GLP-1(7-36)amide (active form) for 4 weeks remarkably reduced the surface areas of the atherosclerotic lesions ( $p<0.0001$; Fig. 1c, s), and suppressed the atheromatous plaque size $(p<0.005$; Fig. 1i, t) and macrophage infiltration ( $p<0.05$; Fig. $10, \mathrm{u})$ in the aortic root. The suppressive effects of GLP-1(7-36)amide were significantly cancelled by simultaneous infusion with exendin(9-39) (Fig. 1d, j, p). Exendin(9-39) alone had no significant effects (Fig. 1e, k, q). Infusion of GLP-1(9-36) amide (inactive form) did not significantly suppress atherosclerotic lesions and macrophage accumulation (Fig. 1f, 1, r).

Infusion of GIP(1-42) (active form) for 4 weeks also remarkably reduced the surface areas of the atherosclerotic lesions ( $p<0.0001$; Fig. $2 \mathrm{c}, \mathrm{s})$, and suppressed atheromatous plaque size $(p<0.005$; Fig. $2 \mathrm{i}, \mathrm{t})$ and macrophage infiltration $(p<0.05$; Fig. 2o, u) in the aortic root, as compared with controls (Fig. 2b, h, n). The suppressive effects of GIP(1-42) were significantly cancelled by simultaneous infusion with $\left(\right.$ Pro $\left.^{3}\right)$ GIP (Fig. 2d, j, p). $\left(\right.$ Pro $\left.^{3}\right)$ GIP alone had no significant effects (Fig. 2e, k, q). Infusion of GIP(3-42) (inactive form) did not significantly suppress atherosclerotic lesions and macrophage accumulation (Fig. 2f, 1, r).

Foam cell formation and related gene expression in exudate peritoneal macrophages from Apoe $e^{-/}$mice The number of exudate peritoneal cells did not differ significantly among all groups of Apoe ${ }^{-/-}$mice administered (Table 1). No remarkable differences in morphological cell characteristics were observed among all groups. As shown in Fig. 3a, oxidised LDL-induced cholesteryl ester accumulation was significantly decreased in macrophages from the GLP-1(736)amide and GIP(1-42) groups, namely by $45 \%$ and $24 \%$ vs controls, respectively $(p<0.0001, p<0.05)$. Infusion with GLP-1(7-36)amide or GIP(1-42) for 4 weeks significantly suppressed CD36 and ACAT-1 protein levels in macrophages $(p<0.005$ and $p<0.05$, respectively; Fig. 3b, c), without affecting levels of ABCA1, ABCG1 and SR-BI (Fig. 3d-f).

Characteristics and laboratory data Table 1 shows characteristics and laboratory data from the selected groups in which GLP-1(7-36)amide and GIP(1-42) exerted antiatherogenic effects, and from vehicle control. Food intake, body weight, systolic blood pressure and plasma concentrations of total cholesterol, HDL-cholesterol and triacylglycerol did not differ among the groups. Plasma active GLP-1 concentrations were significantly higher in the GLP-1(7-36)amide group. Plasma total GIP concentrations were significantly higher in the $\operatorname{GIP}(1-42)$ group. Plasma NEFA concentrations were significantly lower in the GLP-1(7-36)amide and GIP(1-42) groups. Neither glucose nor insulin differed significantly among the groups. In addition, insulin sensitivity showed no significant differences among the groups (electronic supplementary material [ESM] Fig. 1). 

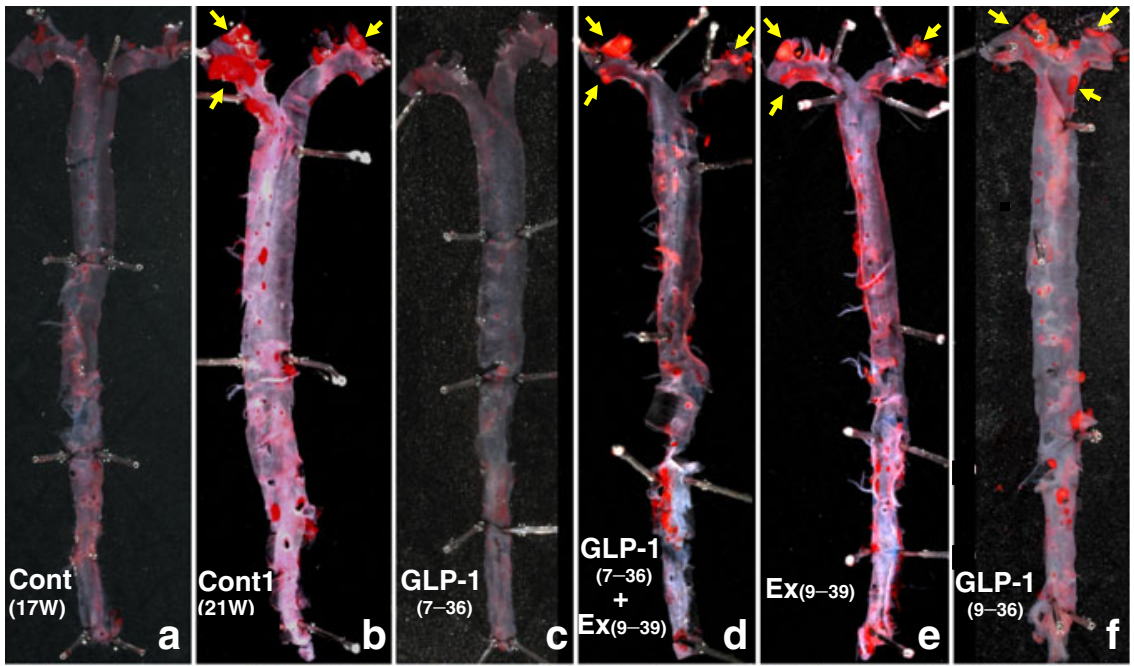

$\mathbf{S}$
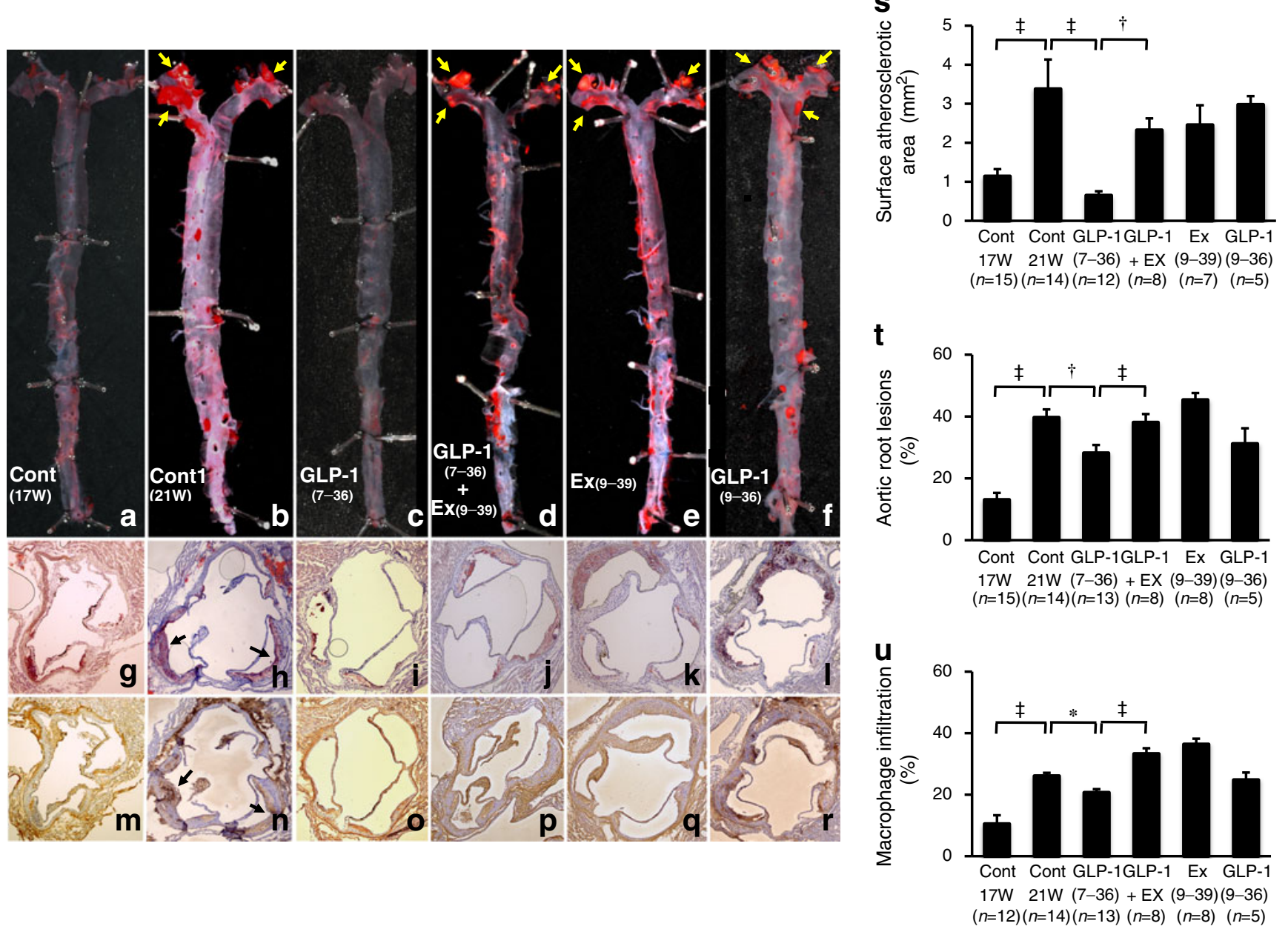

Fig. 1 Suppressive effects of GLP-1 on atherosclerotic lesion development in Apoe $^{-/-}$mice. Apoe ${ }^{-/-}$mice aged 17 weeks $(n=48)$ were infused by osmotic mini-pumps for 4 weeks. Infusions were: vehicle (control [Cont]), GLP-1(7-36)amide, exendin(9-39) (Ex), GLP-1(7-36)amide+exendin(9-39) or GLP-1(9-36)amide. At 21 weeks of age $(21 \mathrm{~W})$ the 48 mice were killed, as were $15 \mathrm{Apoe}^{-/-}$ mice at 17 weeks of age $(17 \mathrm{~W})$ (before infusion). a-f The aortic surface was stained with oil red $\mathrm{O}$. Yellow arrows show notable atherosclerotic lesions. Cross-sections of the aortic root were stained with oil red $\mathrm{O}(\mathbf{g}-\mathbf{l})$ or anti-MOMA-2 antibody $(\mathbf{m}-\mathbf{r})$. Black arrows show notable atheromatous plaques. $\mathbf{s}$ The surface atherosclerotic area, (t) the ratio occupied by atherosclerotic lesions and (u) the ratio occupied by macrophage infiltration in the aortic wall were evaluated. ${ }^{*} p<0.05,{ }^{\dagger} p<0.005$ and ${ }^{\ddagger} p<0.0001$
GLP-1 and GIP receptors in macrophages and arterial $S M C s$ Real-time RT-PCR analyses revealed appreciable levels of Glplr and Gipr mRNA in exudate peritoneal macrophages, compared with the levels found in brain, liver, pancreas, aorta and adipose tissues from non-treated Apoe ${ }^{-/-}$mice (Fig. 4a, b). As shown in Fig. 4c, d, western blotting analyses confirmed the abundant levels of these receptors in mouse macrophages as well as in mouse aortic SMCs and mouse pancreatic islet cells (Primary Cell, Sapporo, Japan). Immunochemical analyses confirmed the abundance of GLP-1R and GIPR in exudate peritoneal macrophages from Apoe $e^{-/-}$mice (Fig. 4e).

Interestingly, we found remarkable changes in expression of GLPIR and GIPR mRNA in THP1 cells and THP1-derived macrophages (Fig. 4f, g). Both receptors were expressed at far higher levels in THP1 cells than in THP1-derived macrophages. The expression of GIPR mRNA in human monocytes was far higher than in human macrophages and human coronary artery SMCs (Lonza) (Fig. 4h).

In vitro effects of incretins on macrophage foam cell formation Exudate peritoneal macrophages obtained from non-treated $\mathrm{Apoe}^{-/-}$mice were incubated for $48 \mathrm{~h}$ with indicated concentrations of GLP-1(7-36)amide, GLP-1(936)amide, GIP(1-42) or GIP(3-42), along with oxidised LDL $(10 \mu \mathrm{g} / \mathrm{ml})$. Exendin(9-39), a GLP-1R antagonist, and $\left(\right.$ Pro $\left.^{3}\right)$ GIP, a GIPR antagonist, were added $1 \mathrm{~h}$ before addition of GLP-1(7-36)amide and GIP(1-42), respectively. GLP-1(7-36)amide (5 nmol/l) or GIP(1-42) (1 nmol/l) 

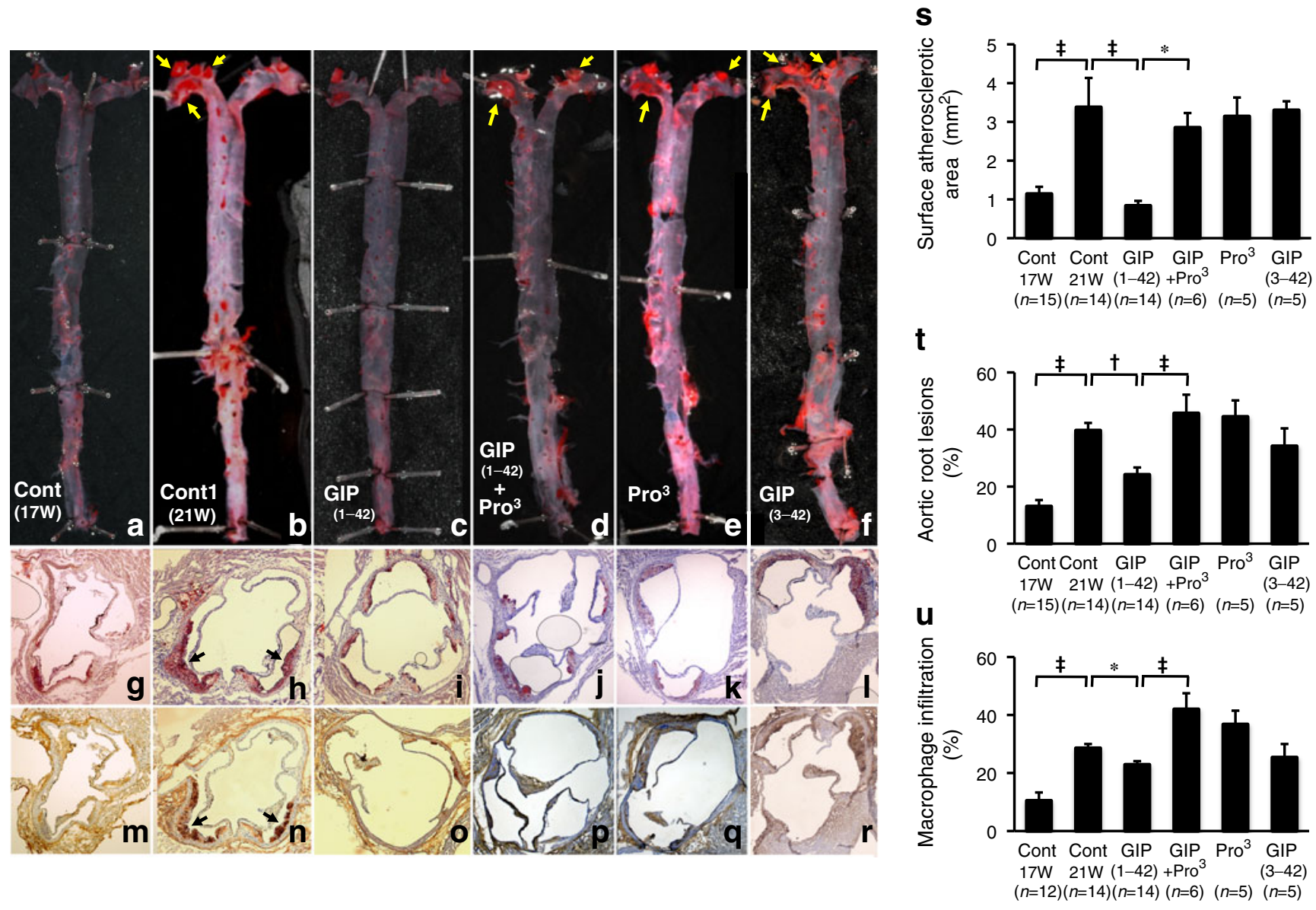

Fig. 2 Suppressive effects of GIP on atherosclerotic lesion development in Apoe $^{-/-}$mice. Apoe $e^{-/-}$mice aged 17 weeks $(n=44)$ were infused by osmotic mini-pumps for 4 weeks. Infusions were: vehicle (control [Cont]), GIP $(1-42)$, $\left(\right.$ Pro $\left.^{3}\right)$ GIP, GIP $(1-42)+\left(\right.$ Pro $\left.^{3}\right)$ GIP or GIP (3-42). At 21 weeks of age ( $21 \mathrm{~W})$, these 44 mice were killed, as were 15 Apo $^{-/-}$mice at 17 weeks of age (17 W) (before infusion). a-f The aortic surface was stained with oil red O. Yellow arrows show remarkable atherosclerotic lesions. Cross-sections of the aortic root were stained with oil red $\mathrm{O}(\mathbf{g}-\mathbf{l})$ or anti-MOMA-2 antibody $(\mathbf{m}-\mathbf{r})$. Black arrows show remarkable atheromatous plaques. $\mathbf{s}$ The surface atherosclerotic area, $(\mathbf{t})$ the ratio occupied by atherosclerotic lesions and (u) the ratio occupied by macrophage infiltration in the aortic wall were evaluated. ${ }^{*} p<0.05,{ }^{\dagger} p<0.005$ and ${ }^{\star} p<0.0001$
Table 1 Characteristics and laboratory data

Values are mean \pm SEM ${ }^{\mathrm{a}} p<0.0005$ and ${ }^{\mathrm{b}} p<0.0001$ vs others, ${ }^{c} p<0.0005$ vs control

\begin{tabular}{llll}
\hline Characteristic & Control & GLP-1(7-36) & GIP(1-42) \\
\hline$n$ & 14 & 13 & 13 \\
Food intake (g/day) & $3.33 \pm 0.47$ & $3.58 \pm 0.67$ & $3.41 \pm 0.57$ \\
Body weight (g) & $44.3 \pm 1.4$ & $43.1 \pm 0.9$ & $44.5 \pm 0.9$ \\
Systolic BP (mmHg) & $111 \pm 3$ & $113 \pm 2$ & $107 \pm 2$ \\
GLP-1 (pmol/l) & $1.51 \pm 0.27$ & $6.14 \pm 1.34^{\mathrm{a}}$ & $1.16 \pm 0.27$ \\
GIP (pmol/l) & $9.26 \pm 1.26$ & $15.2 \pm 1.76$ & $69.0 \pm 8.43^{\mathrm{b}}$ \\
Total cholesterol (mmol/l) & $11.5 \pm 0.72$ & $11.1 \pm 0.56$ & $11.5 \pm 0.70$ \\
HDL-cholesterol (mmol/1) & $0.67 \pm 0.08$ & $0.65 \pm 0.09$ & $0.60 \pm 0.11$ \\
Triacylglycerol (mmol/l) & $1.25 \pm 0.13$ & $0.98 \pm 0.12$ & $1.08 \pm 0.11$ \\
NEFA (mEq/l) & $0.91 \pm 0.06$ & $0.67 \pm 0.02^{\mathrm{c}}$ & $0.66 \pm 0.05^{\mathrm{c}}$ \\
Glucose (mmol/l) & $8.33 \pm 0.50$ & $6.06 \pm 0.78$ & $6.28 \pm 0.72$ \\
Insulin (pmol/l) & $212 \pm 30$ & $268 \pm 71$ & $254 \pm 43$ \\
Exudate peritoneal cells $\left(\times 10^{6}\right)$ & $9.03 \pm 1.86$ & $8.13 \pm 1.47$ & $6.68 \pm 1.78$ \\
\hline
\end{tabular}


Fig. 3 Foam cell formation and protein abundance of $\mathrm{CD} 36$, ACAT-1, ABCA1, ABCG1 and SR-BI in exudate peritoneal macrophages from 43 Apoe $^{-/}$ mice infused with vehicle (control), GLP-1(7-36) or GIP (1-42). a Foam cell formation was evaluated by oxidised LDLinduced cholesteryl ester accumulation in macrophages. Control $15.2 \pm 3.0 \mathrm{nmol} / \mathrm{mg}$ of cell protein. b Western blotting analyses of CD36, (c) ACAT-1, (d) ABCA1, (e) ABCG1 and (f) SR-BI abundance in macrophages. Blots (b-f) show representative results for one of each molecules; bar graphs show results from densitometric analysis of immunoblotting after normalisation with $\beta$-actin. ${ }^{*} p<0.05,{ }^{\dagger} p<0.005$ and ${ }^{\star} p<0.0001$ a

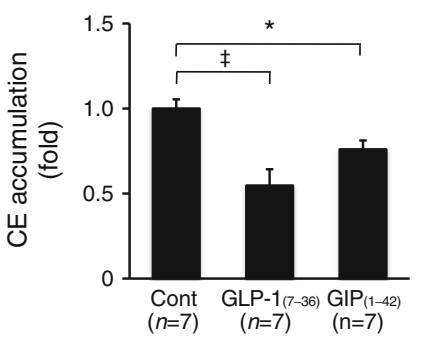

d

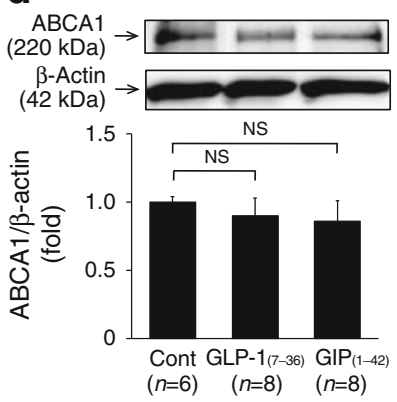

b

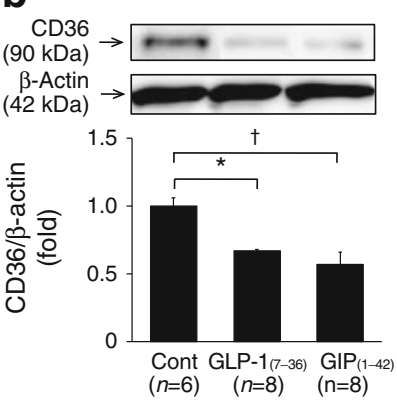

e

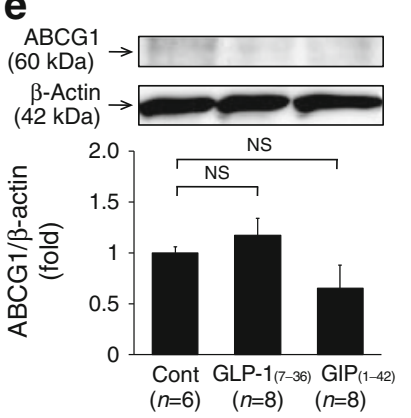

C

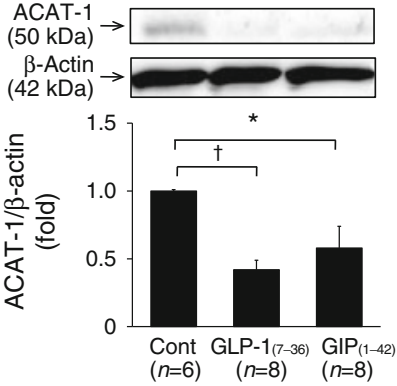

f

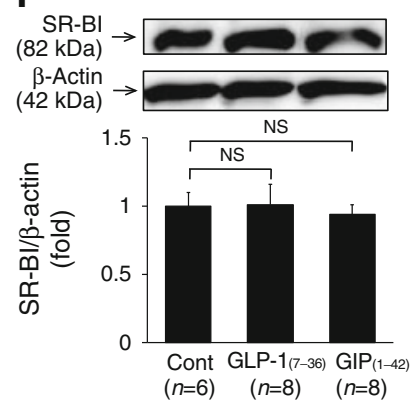

(active forms) significantly reduced cholesteryl ester accumulation $(p<0.05$ for both; Fig. 5a, b). The maximal reductions in cholesteryl ester accumulation by GLP-1(736)amide $(5 \mathrm{nmol} / \mathrm{l})$ or $\operatorname{GIP}(1-42)(1 \mathrm{nmol} / \mathrm{l})$ were significantly abolished by pretreatment with exendin(939) $(50 \mathrm{nmol} / \mathrm{l})$ and $\left(\mathrm{Pro}^{3}\right) \mathrm{GIP}(10 \mathrm{nmol} / \mathrm{l})$, respectively $(p<$ 0.05 for both; Fig. 5e, f). Both effects of incretins were significantly cancelled by MDL12,330A ( $5 \mu \mathrm{mol} / \mathrm{l})$, an adenylate cyclase inhibitor, and mimicked by forskolin $(10 \mu \mathrm{mol} / \mathrm{l})$, an adenylate cyclase activator $(p<0.01, p<$ 0.05 ; Fig. 5g). In contrast, GLP-1(9-36)amide and GIP(342) (inactive forms) had no significant effect on cholesteryl ester accumulation (Fig. 5c, d).

In vitro effects of incretins on atherogenic gene expression in aortic endothelial cells, and aortic SMC proliferation GLP-1 (7-36)amide (10 nmol/l) and GIP(1-42) (5 nmol/l) significantly suppressed expression of $M C P 1, V C A M 1, I C A M 1$ and $P A I 1$ in human aortic endothelial cells ( $p<0.05$ at least; Fig. 6a).

GLP-1(7-36)amide (10 nmol/l) or GIP(1-42) $(1 \mathrm{nmol} / \mathrm{l})$ along with PKF275-055 (20 $\mu \mathrm{mol} / \mathrm{l})$ significantly suppressed the proliferation of human aortic SMCs $(p<0.01, p<0.05$; Fig. 6b, c), without cytotoxic effects (Fig. 6d).

\section{Discussion}

This study is the first to demonstrate a variety of antiatherogenic effects of GLP-1 and GIP on vascular cells occurring independently of glucose-lowering activity. Active

forms of GLP-1 and GIP suppressed production of monocyte chemoattractant and adhesion molecules in aortic endothelial cells, monocyte/macrophage infiltration into the aortic wall, macrophage foam cell formation and aortic SMC proliferation, helping to prevent atherosclerotic lesion development in Apoe $^{-/-}$mice. However, the blockade of GLP-1R and GIPR with the specific receptor antagonists alone did not affect the development of atherosclerotic lesions, suggesting that endogenous GLP-1 and GIP levels are far too low to counteract the development of atherosclerosis in this specific animal model.

Several lines of evidence suggest that GLP-1 and its receptor agonists, but not GIP, have vasoprotective effects $[1,2]$. GLP-1 and exendin-4 have a protective effect on oxidative stress-induced DNA damage in HUVECs [17]. Exendin-4 suppresses high glucose-induced TNF- $\alpha$ and IL$1 \beta$ levels in THP1 cells, and TNF- $\alpha$-induced ICAM-1 production in human glomerular microvascular endothelial cells [18]. Exenatide (synthetic exendin-4) reduces intimal hyperplasia in balloon-injured carotid arteries of insulinresistant Zucker fatty rats [19]. Arakawa et al. [5] recently reported that exendin-4 attenuates atherosclerotic lesions in Apoe $^{-/-}$mice and suppresses monocyte/macrophage adhesion on the arterial wall by inhibiting the inflammatory response in macrophages. Their report is the initial demonstration of an anti-atherogenic action of one of the incretin agents. However, the effects of native GLP-1 and GIP on atherosclerosis have yet to be determined. Our findings here are the first to show a potent suppression of atherosclerotic lesions by long-term infusion of native incretins into Apoe ${ }^{-/-}$mice. We demonstrated that GLP-1 


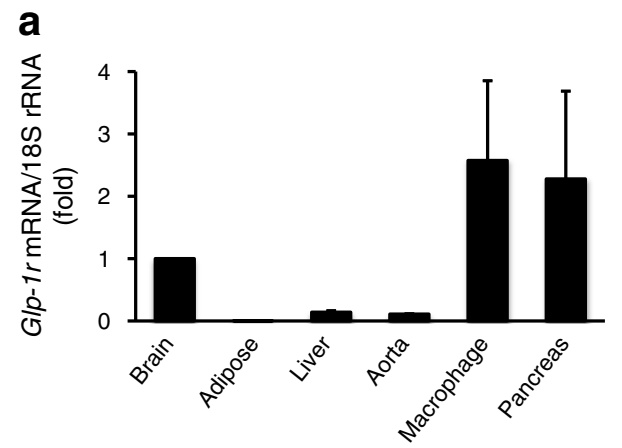

\section{C}
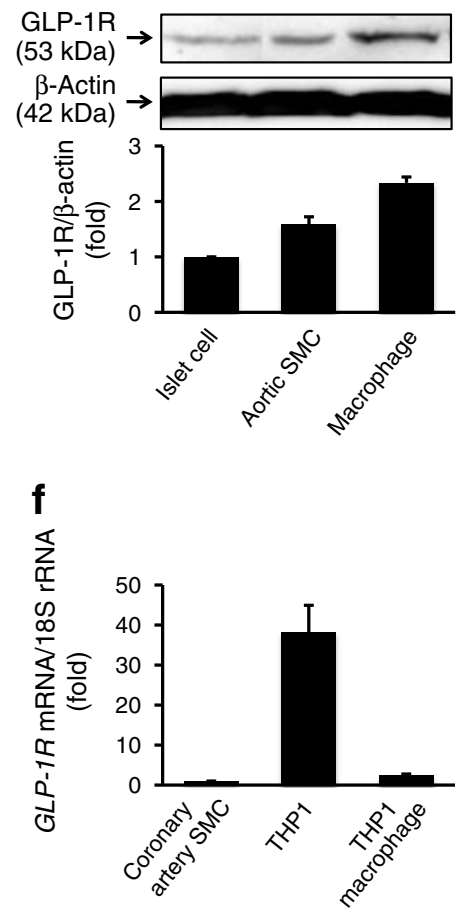

b

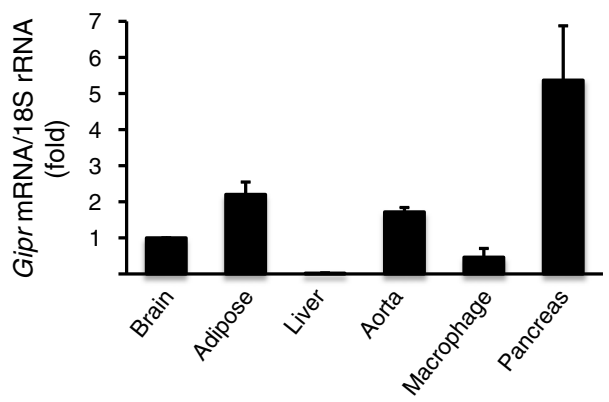

d

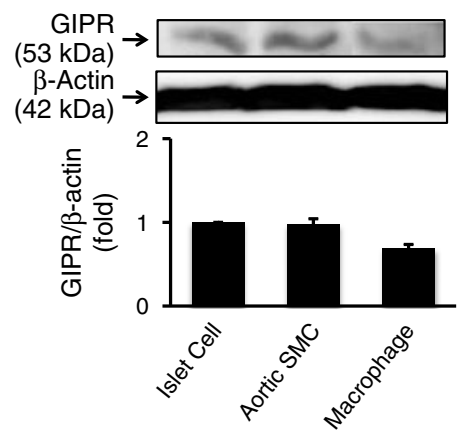

g

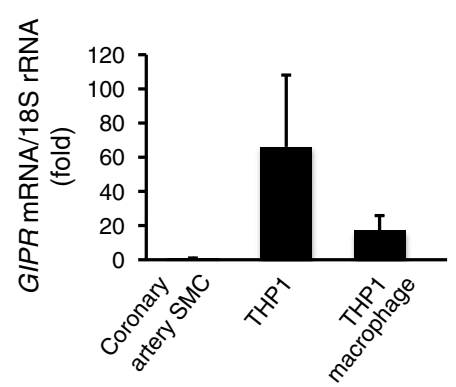

e

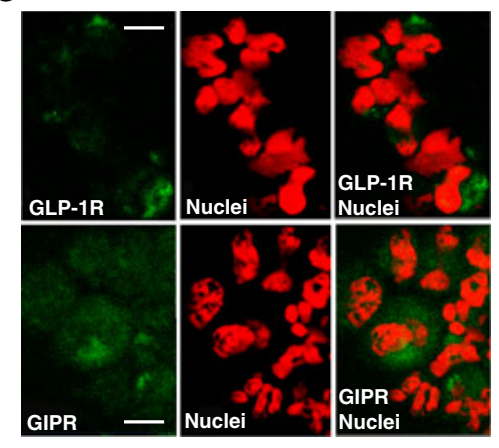

h

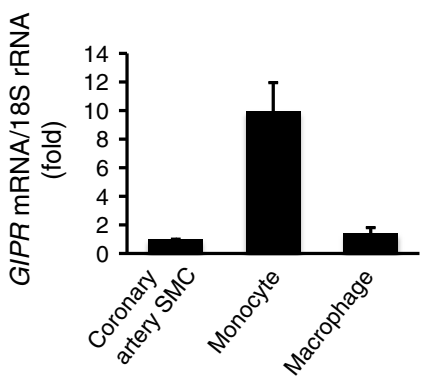

Fig. 4 Expression and abundance of GLP-1R and GIPR in monocytes/macrophages and arterial SMCs. a Glp1r and (b) Gipr mRNA in exudate peritoneal macrophages and tissues as labelled from nontreated $A$ poe ${ }^{-/-}$mice were measured by real-time RT-PCR. c Western blotting analyses of GLP-1R and (d) GIPR abundance in exudate peritoneal macrophages of non-treated $A p o e^{-/-}$mice and other mouse tissues as labelled. Three independent experiments were performed. e Immunostaining of GLP-1R or GIPR in exudate peritoneal macro-

and GIP directly suppressed the expression of $M C P 1$, VCAM1, ICAM1 and PAI1 in aortic endothelial cells, as well as suppressing aortic SMC proliferation and macrophage foam cell formation associated with the downregulation of CD36 and ACAT-1.

GLP-1R and GIPR, which belong to the seventransmembrane domain family of receptors, were produced in Apoe $e^{-1-}$ mouse macrophages. Our in vitro study showed that the suppressive effects of active forms of GLP-1 and GIP on macrophage foam cell formation were abolished by specific antagonists of GLP-1R and GIPR. phages from non-treated Apoe $e^{-/-}$mice. Green, GLP-1R or GIPR as indicated; red, nuclei; red + green, overlay of GLP-1R or GIPR and nuclei. Scale bars $50 \mu \mathrm{m}$. f GLPIR and (g) GIPR mRNA levels in human coronary artery SMCs, THP1 cells and THP1-derived macrophages. h GIPR mRNA level in human monocytes and monocytederived macrophages were measured by real-time RT-PCR. Results were obtained from three to six independent experiments

These findings indicate that active GLP-1 and GIP suppress macrophage foam cell formation through GLP$1 \mathrm{R}$ and GIPR, respectively. Compared with GIP, the somewhat stronger actions of GLP-1 in the suppression of foam cell formation may depend on the greater abundance of GLP-1R in macrophages. The in vivo effects of GLP-1 and GIP on foam cell formation in exudate peritoneal macrophages from $A p o e^{-/-}$mice were much more powerful than these suppressive effects in vitro. We elucidated these discrepancies by demonstrating changes in the expression of both receptors using primary 

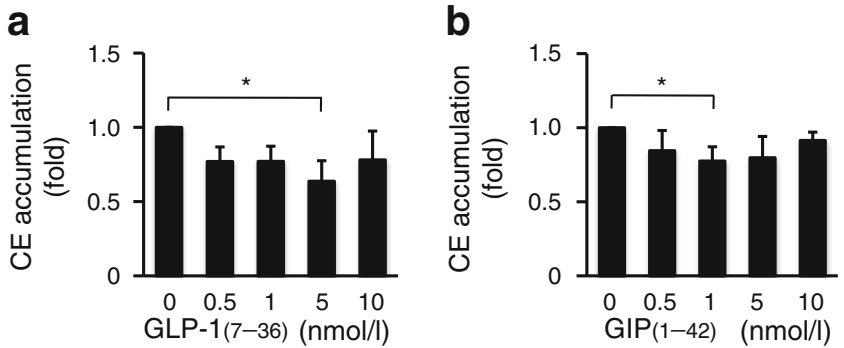
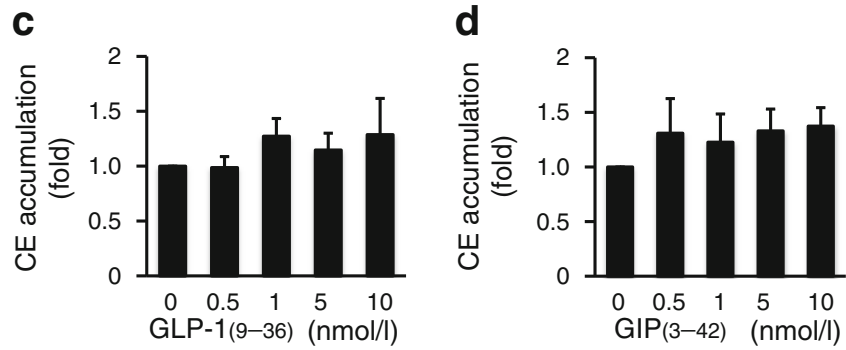

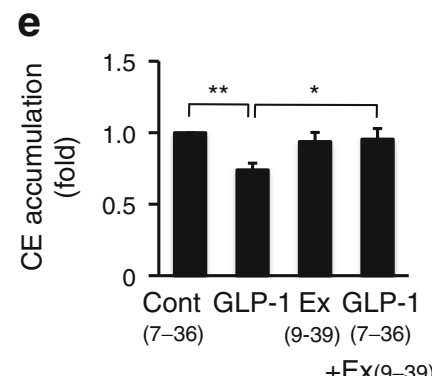

$+\mathrm{Ex}(9-39)$

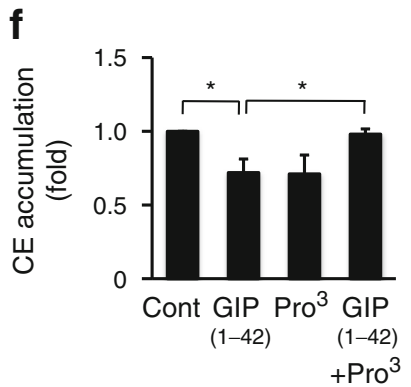

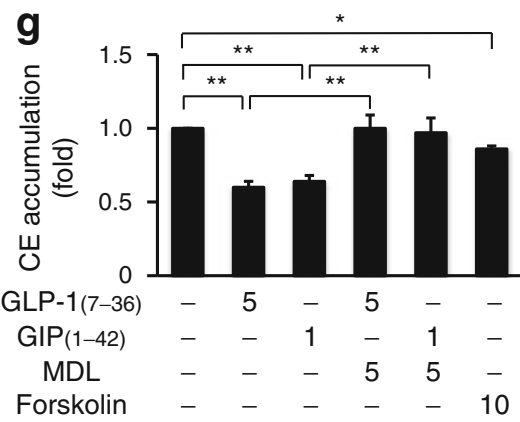

Fig. 5 In vitro suppressive effects of incretins as indicate on foam cell formation in exudate peritoneal macrophages, expressed as cholesteryl ester (CE) accumulation, from non-treated $A p o e^{-/-}$mice $(\mathbf{a}-\mathbf{g})$. The doses of reagents are described in the Results section. Data were

human monocytes and THP1 cells. The expression of both receptors decreased during the differentiation from monocytes into macrophages. Thus, monocytes/macrophages during differentiation in vivo may be more sensitive to obtained from three to six independent experiments. Control $4.0 \pm 1.3$ (a, b), $5.2 \pm 2.2(\mathbf{c}, \mathbf{d}), 4.9 \pm 0.4(\mathbf{e}, \mathbf{f})$ and $13.6 \pm 0.6 \mathrm{nmol} / \mathrm{mg}$ of cell protein (g). ${ }^{*} p<0.05,{ }^{* *} p<0.01$. Ex, exendin; MDL12,330A, adenylate cyclase inhibitor

incretins than cultured macrophages obtained after differentiation in untreated Apoe $e^{-/-}$mice, a difference that could be due to the high levels of the two incretin receptors in monocytes.
Fig. 6 Expression of $M C P 1$ (white), VCAM1 (oblique), ICAM1 (chequer) and PAI1 (black) in human aortic endothelial cells (a). b, c Human aortic SMC proliferation under treatments as labelled. Data were obtained from three to four independent experiments; ${ }^{*} p<$ $0.05, * * p<0.01,{ }^{\dagger} p<0.005$. d Phalloidin-TRITC/DAPI staining showing actin cytoskeleton and nuclear morphology of human aortic SMCs with or without treatments as labelled. Scale bars $50 \mu \mathrm{m}$. Doses of reagents used are described in the Results section. PKF275-055 (vildagliptin analogue), DPP-4 inhibitor
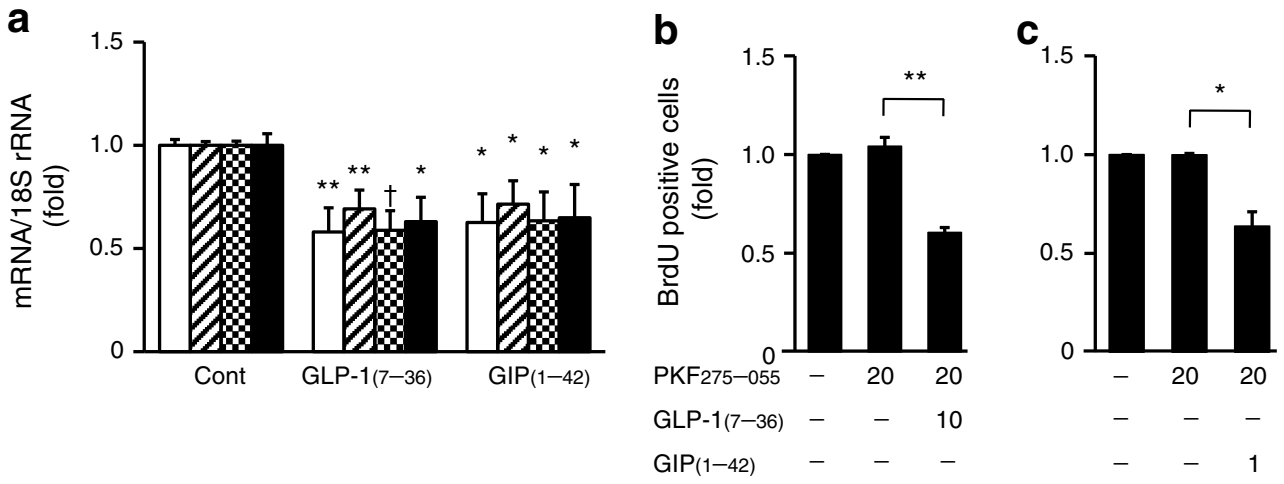
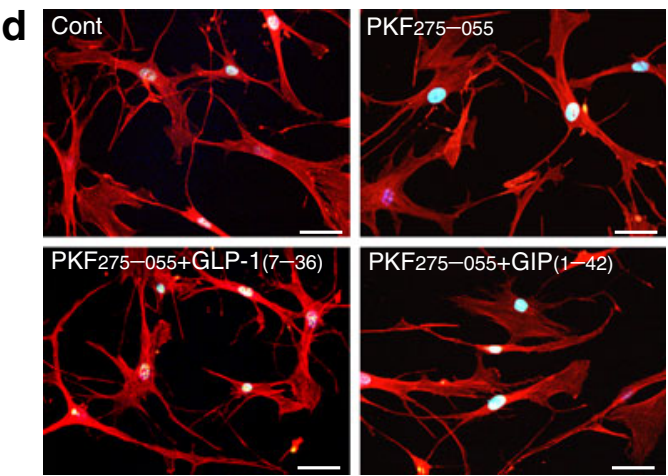
Activation of GLP-1R and GIPR stimulates the production of cAMP via adenylate cyclase action [4], whereupon cAMP signalling is further amplified and diversified via activation of several downstream factors, such as protein kinase A (PKA), phosphatidylinositol-3-kinase (PI3K), protein kinase $\mathrm{C}(\mathrm{PKC})$ and extracellular signal-regulated kinase (ERK) 1/2 [1, 4]. Arakawa et al. [5] reported that exendin-4 suppresses the lipopolysaccharide-induced mRNA expression of $T N F-\alpha$ (also known as $T N F$ ) and $M C P 1$ via the cAMP/PKA pathway. We showed that suppressive effects of GLP-1 and GIP on macrophage foam cell formation were cancelled by the adenylate cyclase inhibitor and mimicked by the adenylate cyclase activator. These findings indicate that both incretins suppress macrophage foam cell formation via cAMP. We also tried to determine the signal transductions involved in the downregulation of CD36 and ACAT-1 by incretins. Previous studies have shown that CD36 and ACAT-1 levels were unrelated to cAMP and PKA [20-22], but related to PI3K, PKC and ERK [23, 24]. However, incretins-induced CD36 and ACAT-1 downregulation was not wholly abolished by specific inhibitors of adenylate cyclase, PI3K, PKA, ERK (ESM Fig. 2) or PKC (data not shown). Future studies are needed to clarify the complex networks of unidentified signalling pathways concerned.

The doses for the incretin infusions in the present study were the same as those usually used in studies by others $[11,12]$. Plasma GIP concentrations were sevenfold higher in Apoe $^{-/-}$mice infused with GIP vs vehicle-infused controls, whereas GLP-1 concentrations were only fourfold higher than the control level in Apoe ${ }^{-/-}$mice infused with GLP-1. GLP-1 was measured by an ELISA kit that is only capable of detecting active GLP-1, while the GIP test kit measured active and inactive forms of GIP. The increased plasma levels of GLP-1 and GIP are comparable to the average physiological concentrations of incretins after a meal tolerance test in humans [25].

The present study is the first to provide evidence that incretin-based treatments using native GLP-1 and GIP help to prevent development of atherosclerotic lesions. This may open up a new therapeutic window for the treatment of atherosclerosis and related diseases. Extensive clinical studies will be required to elucidate whether incretinbased therapies, above and beyond glycaemic benefits, are favourable for preventing atherosclerosis in type 2 diabetes.

Acknowledgements This work was presented in part at the Scientific Sessions of the American Heart Association, Chicago, IL, 13-17 November 2010, the European Association for the Study of Diabetes, Stockholm, Sweden, 19-24 September 2010, and the American Diabetes Association, Orlando, FL, 25-29 June 2010. It was supported in part by a Grant-in-Aid for Scientific Research (C) ( 22590831 to T. Watanabe) from the Japan Society for the Promotion of Science.
Contribution statement All authors were responsible for (1) conception and design, or analysis and interpretation of data; (2) drafting the article or revising it critically for important intellectual content; and (3) final approval of the version to be published.

Duality of interest The authors declare that there is no duality of interest associated with this manuscript.

Open Access This article is distributed under the terms of the Creative Commons Attribution Noncommercial License which permits any noncommercial use, distribution, and reproduction in any medium, provided the original author(s) and source are credited.

\section{References}

1. Kim W, Egan JM (2008) The role of incretins in glucose homeostasis and diabetes treatment. Pharmacol Rev 60:470-512

2. Drucker DJ (2006) The biology of incretin hormones. Cell Metab 3:153-165

3. Nystrom T, Gutniak MK, Zhang Q et al (2004) Effects of glucagon like peptide on endothelial function in type 2 diabetes patients with stable coronary artery disease. Am J Physiol Endocrinol Metab 287:E1209-E1215

4. Hansotia T, Drucker DJ (2005) GIP and GLP-1 as incretin hormones: lessons from single and double incretin receptor knockout mice. Regul Peptides 128:125-134

5. Arakawa M, Mita T, Azuma K et al (2010) Inhibition of monocyte adhesion to endothelial cells and attenuation of atherosclerotic lesion by a glucagon-like peptide-1 receptor agonist, exendin-4. Diabetes 59:1030-1037

6. Goto H, Nomiyama T, Mita T et al (2011) Exendin-4, a glucagonlike peptide-1 receptor agonist, reduces intimal thickening after vascular injury. Biochem Biophys Res Commun 405:79-84

7. Liu H, Dear AE, Knudsen LB, Simpson RW (2009) A long-acting glucagon-like peptide-1 analogue attenuates induction of plasminogen activator inhibitor type-1 and vascular adhesion molecules. J Endocrinol 201:59-66

8. Matsui T, Nishino Y, Takeuchi M, Yamagishi S (2011) Vildagliptin blocks vascular injury in the thoracic aorta of diabetic rats by suppressing advanced glycation end product-receptor axis. Pharmacol Res 63:383-388

9. Saha P, Modarai B, Humphries J et al (2009) The monocyte/ macrophage as a therapeutic target in atherosclerosis. Curr Opin Pharmacol 9:109-118

10. Xu G, Watanabe T, Iso $Y$ et al (2009) Preventive effects of heregulin- $\beta_{1}$ on macrophage foam cell formation and atherosclerosis. Circ Res 105:500-510

11. Zhang J, Tokui Y, Yamagata K et al (2007) Continuous stimulation of human glucagon-like peptide-1(7-36)amide in a mouse model (NOD) delays onset of autoimmune type 1 diabetes. Diabetologia 50:1900-1909

12. McClean PL, Irwin N, Hunter K, Gault VA, Flatt PR (2008) $\left(\right.$ Pro $\left.^{3}\right)$ GIP[mPEG]: novel, long-acting, mPEGylated antagonist of gastric inhibitory polypeptide for obesity-diabetes (diabesity) therapy. $\mathrm{Br}$ J Pharmacol 155:690-701

13. Shiraishi Y, Watanabe T, Suguro T et al (2008) Chronic urotensin II infusion enhances macrophage foam cell formation and atherosclerosis in apolipoprotein E-knockout mice. J Hypertens 26:1955-1965

14. Nagashima M, Watanabe T, Shichiri $M$ et al (2010) Chronic infusion of salusin- $\alpha$ and $-\beta$ exerts opposite effects on macro- 
phage foam cell formation and atherosclerosis in apolipoprotein E-deficient mice. Atherosclerosis 212:70-77

15. Watanabe T, Nishio K, Kanome T et al (2008) Impact of salusin- $\alpha$ and $-\beta$ on human macrophage foam cell formation and coronary atherosclerosis. Circulation 117:638-648

16. Palmieri FE, Ward PE (1989) Dipeptidyl(amino)peptidase IV and post proline cleaving enzyme in cultured endothelial and smooth muscle cells. Adv Exp Med Biol 247A:305-311

17. Oeseburg H, de Boer RA, Buikema H, van der Harst P, van Gilst WH, Silljé HH (2010) Glucagon-like peptide 1 prevents reactive oxygen species-induced endothelial cell senescence through the activation of protein kinase A. Arterioscler Thromb Vasc Biol 30:1407-1414

18. Kodera R, Shikata K, Kataoka HU et al (2011) A glucagon-like peptide-1 receptor agonist ameliorates renal injury through its anti-inflammatory action without lowering blood glucose level in a rat model of type 1 diabetes. Diabetologia 54:965-978

19. Murthy SN, Hilaire RC, Casey DB et al (2010) The synthetic GLP-1 receptor agonist, exenatide, reduces intimal hyperplasia in insulin resistant rats. Diab Vasc Dis Res 7:138-144
20. Feng J, Han J, Pearce FA et al (2000) Induction of CD36 expression by oxidized LDL and IL-4 by a common signaling pathway dependent on protein kinase C and PPAR- $\gamma$. J Lipid Res 41:688-696

21. Okutsu R, Yoshikawa T, Nagasawa M et al (2009) Cilostazol inhibits modified low-density lipoprotein uptake and foam cell formation in mouse peritoneal macrophages. Atherosclerosis 204:405-411

22. Botham KM (1992) Cyclic AMP and the regulation of cholesterol metabolism. Biochem Soc Trans 20:454-459

23. Watanabe T, Suguro T, Kanome T et al (2005) Human urotensin II accelerates foam cell formation in human monocyte-derived macrophages. Hypertension 46:738-744

24. Hongo S, Watanabe T, Arita S et al (2009) Leptin modulates ACAT1 expression and cholesterol efflux from human macrophages. Am J Physiol Endocrinol Metab 297:E474-E482

25. Lee S, Yabe D, Nohtomi K, Morita R, Seino Y, Hirano T (2010) Intact glucagon-like peptide-1 levels are not decreased in Japanese patients with type 2 diabetes. Endocr J 57:119-126 